\title{
Red giant branch stars as probes of stellar populations
}

\section{Properties of the newly discovered globular cluster GLIMPSE-C01}

\author{
V. D. Ivanov ${ }^{1}$, R. Kurtev ${ }^{2}$, and J. Borissova ${ }^{1}$ \\ 1 European Southern Observatory, Ave. Alonso de Cordova 3107, Casilla 19, Santiago 19001, Chile \\ e-mail: [vivanov; jborisso]@eso.org \\ 2 Departamento de Física y Meteorología, Facultad de Ciencias, Universidad de Valparaíso, Av. Gran Bretaña 644, \\ Playa Ancha, Casilla 53, Valparaíso, Chile \\ e-mail: rkurtev@uv.cl
}

Received 19 March 2005 / Accepted 26 May 2005

\section{ABSTRACT}

Deep near infrared photometry of the newly discovered Galactic globular Cluster GLIMPSE-C01 is reported. We derived for the first time the metal abundance of this object from the slope of the RGB: $[\mathrm{Fe} / \mathrm{H}]=-1.61 \pm 0.14$ in the scale of Zinn (as implemented in Harris 1996), $[\mathrm{Fe} / \mathrm{H}]=-1.44 \pm 0.12$ in the scale of Caretta \& Gratton $(1997)$, and $[\mathrm{Fe} / \mathrm{H}]=-1.12 \pm 0.12$ in the scale of Ferraro et al. (1999). The tip and the clump of the red giant branch were used to confirm the estimates of Kobulnicky et al. (2005), placing the cluster at $D \sim 3.7 \pm 0.8 \mathrm{kpc}$, behind $A_{\mathrm{V}} \sim 15$ mag of visual extinction. The best fit to the radial surface brightness profile with a single-mass King's model yielded a core radius $r_{\mathrm{c}}=0.78$ arcmin, tidal radius $r_{\mathrm{t}}=27$ arcmin and central concentration $c=1.54$.

Finally, we estimate the number of the "missing" globulars in the central region of the Milky Way. Based on the spatial distribution of the known clusters, and assuming radial symmetry around the Galactic center, we conclude that the Milky Way contains at least $10 \pm 3$ undiscovered objects. The distribution of known clusters in the bulge seem to resemble the orientation of the Milky Way bar.

Key words. Galaxy: globular clusters: general - Galaxy: abundances - stars: distances - Galaxy: bulge - Galaxy: fundamental parameters

\section{Introduction}

The all-sky infrared surveys carried out during recent years have revealed a number of new clusters, hidden by the dust extinction in the plane of the Milky Way. They usually are subjected to $A_{\mathrm{V}} \geq 10-20$ mag of extinction, making them invisible in the optical wavebands. The vast majority of these objects appear to be a few million years old (Ivanov et al. 2002, 2005; Borissova et al. 2003, 2005) but a few have proved to be analogues of "classical" globular clusters (Hurt et al. 2000; Kobulnicky et al. 2005; Carraro 2005).

The globular cluster GLIMPSE-C01 was discovered (Kobulnicky et al. 2005) during the Galactic Legacy Infrared Mid-Plane Survey Extraordinaire (hereafter GLIMPSE; Benjamin et al. 2003). The survey is mapping the Galactic plane from $|l|=10^{\circ}$ to $65^{\circ}$ and $|b| \leq 1^{\circ}$ with the Infrared-Array Camera (IRAC; Fazio et al. 2004) on the Spitzer Space Telescope in 3.6, 4.5, 5.8 and 8.0 micron bands. It was also identified independently by Simpson \& Cotera (2004) who cross-correlated ASCA X-ray and IRAS sources with the 2 MASS.
The isochrone comparison indicated that GLIMPSE-C01 is indeed a globular cluster, at least a few Gyr old. The near infrared color-magnitude diagram (CMD) suggested that the object suffers $A_{\mathrm{V}}=15 \pm 3$ mag of extinction, and the analysis of the line-of-sight ${ }^{13} \mathrm{CO}$ yielded a distance of 3.1-5.2 kpc, based on a kinematic model of the Milky Way. The question of the cluster abundance remained open, as did the need to verify the distance.

Here we report properties - metallicity, extinction, and distance - derived from deep near infrared photometry of GLIMPSE-C01. We also estimate the possible number of undiscovered globular clusters in the central region of the Milky Way.

\section{Observations and data reduction}

The $J H K_{\mathrm{S}}$ imaging observations of GLIMPSE-C01 were carried out in Nov 2004 under non-photometric condition and 1 arcsec seeing with the SofI (Son of ISAAC) at the NTT. The instrument is equipped with Hawaii $\mathrm{HgCdTe} 1024 \times 1024$ detector, with a pixel scale of $0.288 \operatorname{arcsec} \mathrm{px}^{-1}$. 
We took 12 images in each filter, in jittering mode with 3 arcmin jitter box size to ensure that on each image the cluster is located at a different place on the array. Each image was the average of 3 frames of $10 \mathrm{~s}$ for $J, 13$ frames of $3 \mathrm{~s}$ for $H$ and 20 frames of $3 \mathrm{~s}$ for $K_{\mathrm{S}}$, with total integration times of $6,7.8$ and 12 min, respectively. Individual images were sky-subtracted, flat-fielded, aligned and combined into a single image.

The stellar photometry of the final images was carried out with ALLSTAR in DAOPHOT II (Stetson 1993). The typical photometric errors vary from 0.03 mag for the $K_{\mathrm{S}} \sim 10 \mathrm{mag}$ stars to $0.10 \mathrm{mag}$ for $K_{\mathrm{S}} \sim 13$ and $0.15 \mathrm{mag}$ for $K_{\mathrm{S}} \sim 16 \mathrm{mag}$. The photometry of the the faint stars is affected by crowding, especially near the cluster center but our metallicity estimate is based on stars with $K_{\mathrm{S}} \leq 13 \mathrm{mag}$.

The photometric calibration was performed by comparing our instrumental magnitudes with the 2 MASS measurements of 67 stars, covering the color ranges $0.61 \leq J-K_{\mathrm{S}} \leq 5.98$ and $0.21 \leq H-K_{\mathrm{S}} \leq 2.32 \mathrm{mag}$, and magnitude range $8.33 \leq K_{\mathrm{S}} \leq$ $13.13 \mathrm{mag}$. The transformation equations are:

$J-K_{\mathrm{S}}=(0.981 \pm 0.005) \times(j-k)+(3.208 \pm 0.007)$,

$H-K_{\mathrm{S}}=(0.971 \pm 0.016) \times(h-k)+(1.820 \pm 0.014)$,

and

$$
\begin{aligned}
K_{\mathrm{S}}=(1.008 \pm & 0.007) \times k+(0.0126 \pm 0.006) \\
& \times\left(J-K_{\mathrm{S}}\right)-(10.096 \pm 0.131),
\end{aligned}
$$

with rms $=0.047,0.062$ and $0.044 \mathrm{mag}$, respectively. Here $k$, $j-k, h-k$ are the instrumental magnitudes and colors and $K_{\mathrm{S}}$, $J-K_{\mathrm{S}}, H-K_{\mathrm{S}}$ are the magnitudes and colors in the 2 MASS system. The standard error values are given after each coefficient.

A true-color composite of GLIMPSE-C01 is shown in Fig. 1. The image indicates substantial variations of the extinction in the field. Dust structures are present even across the cluster face, suggesting that the completeness of the photometry varies significantly. The crowding also contributes to this effect, as it can be seen from the luminosity function of stars in the field of GLIMPSE-C01 (Fig. 2): it is clear that the completeness of the photometry in the cluster area (long-dashed line) is at least a magnitude shallower than that in the field (dotted line).

\section{Properties of GLIMPSE-C01}

\subsection{Structural parameters}

The structural parameters of GLIMPSE-C01 were determined using the iterative star counts method of King (1962) after randomly removing the field stars (see Sect. 3.3). The radial profile was built only from the stars in the South-Eastern half of the GLIMPSE-C01 to exclude the effects of the variable extinction across the cluster face. The best fit to the radial surface brightness profile with a single-mass King's model (Fig. 3) yielded a core radius $r_{\mathrm{c}}=0.78$ arcmin, tidal radius $r_{\mathrm{t}}=27$ ar$\mathrm{cmin}$, and central concentration $c=1.54$. The inhomogeneous foreground extinction - significant even in the near-infrared makes it impossible to confirm the suggestion of Kobulnicky et al. (2005) that GLIMPSE-C01 is elongated but we cannot exclude such a possibility.

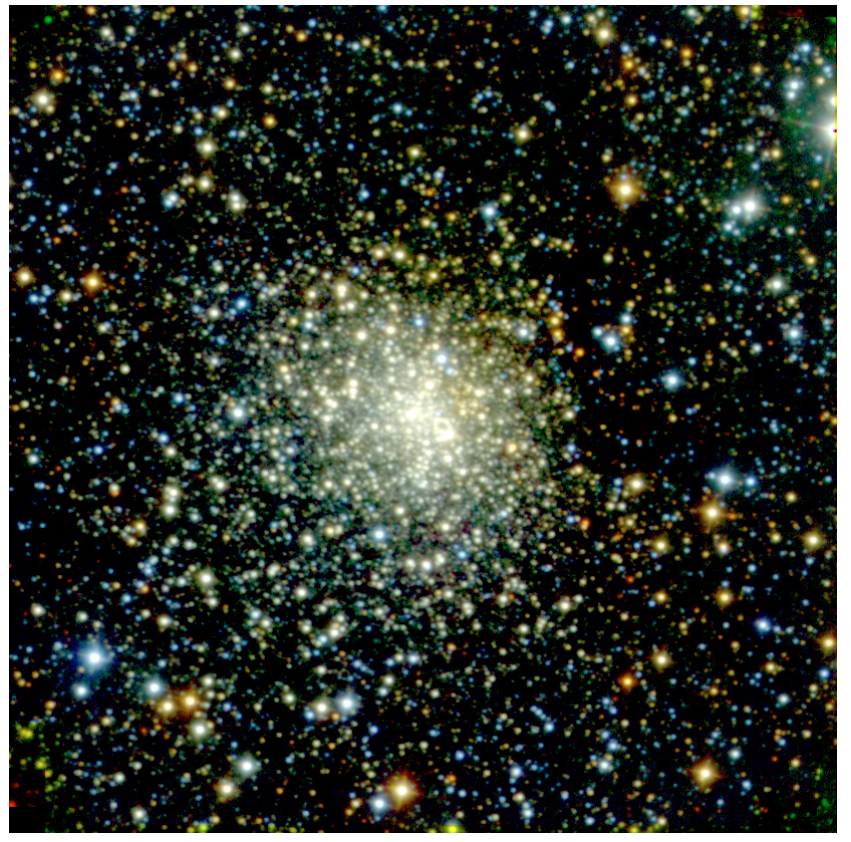

Fig. 1. True-color near infrared composite image of GLIMPSE-C01. The $J, H$, and $K_{\mathrm{S}}$ bands correspond to blue, green and red, respectively. North is up, and East is to the left. The field of view is 4.92 arcmin on a side. The dark lane in the North-West part of the image corresponds to a region of mid-infrared emission, and it is a real feature. (This figure is available in color in the electronic version of the journal.)

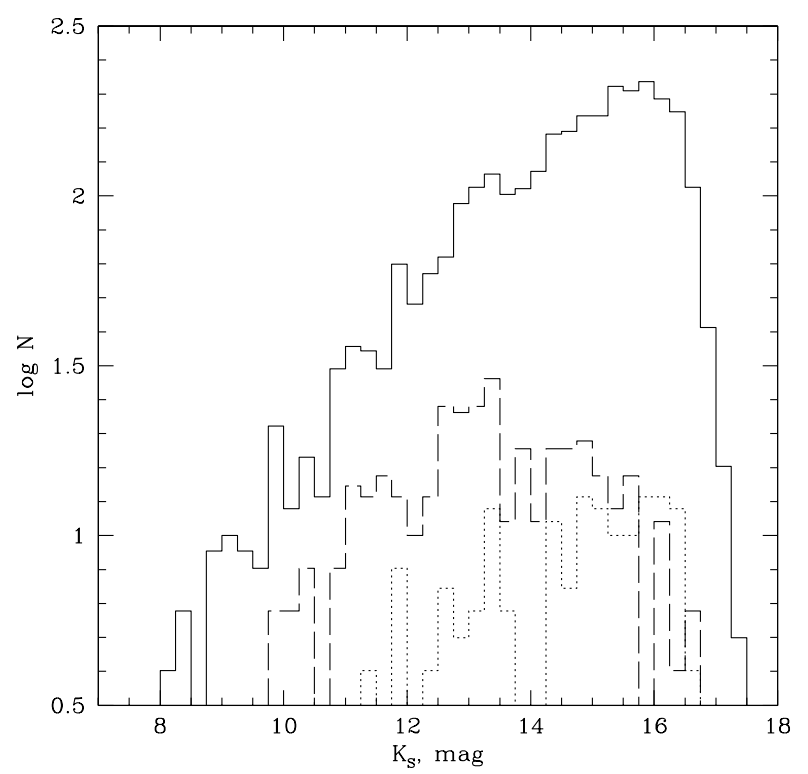

Fig. 2. Near infrared luminosity function for: all stars in the field of GLIMPSE-C01 (solid line), stars within $R \leq 30$ arcsec from the center of the cluster (long-dashed line), and stars in an annulus with inner radius of 50 arcsec and the same area as the cluster region (dotted line).

\subsection{Distance and extinction}

The CMD of GLIMPSE-C01 is plotted in Fig. 4. The left panel contains all stars with $J$ and $K_{\mathrm{S}}$ photometry, and shows the presence of a few sequences. The nature of these sequences 


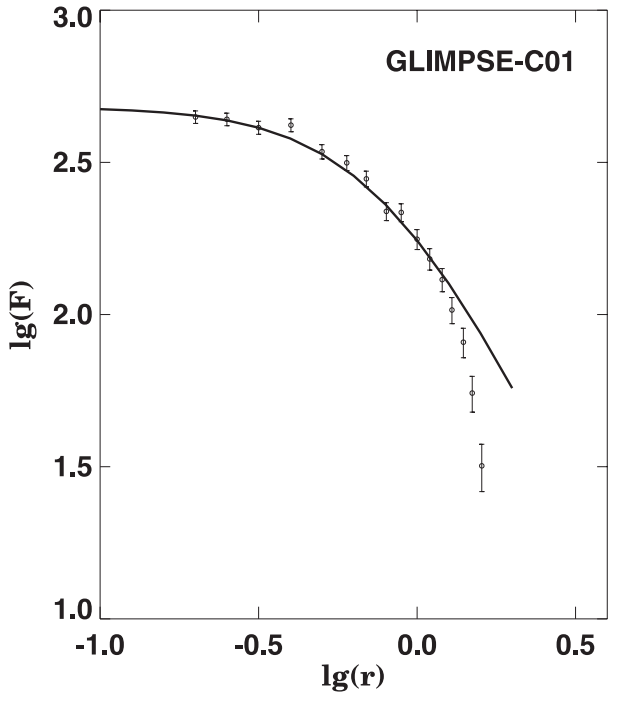

Fig. 3. Structural parameters of the cluster: King's profile fit.

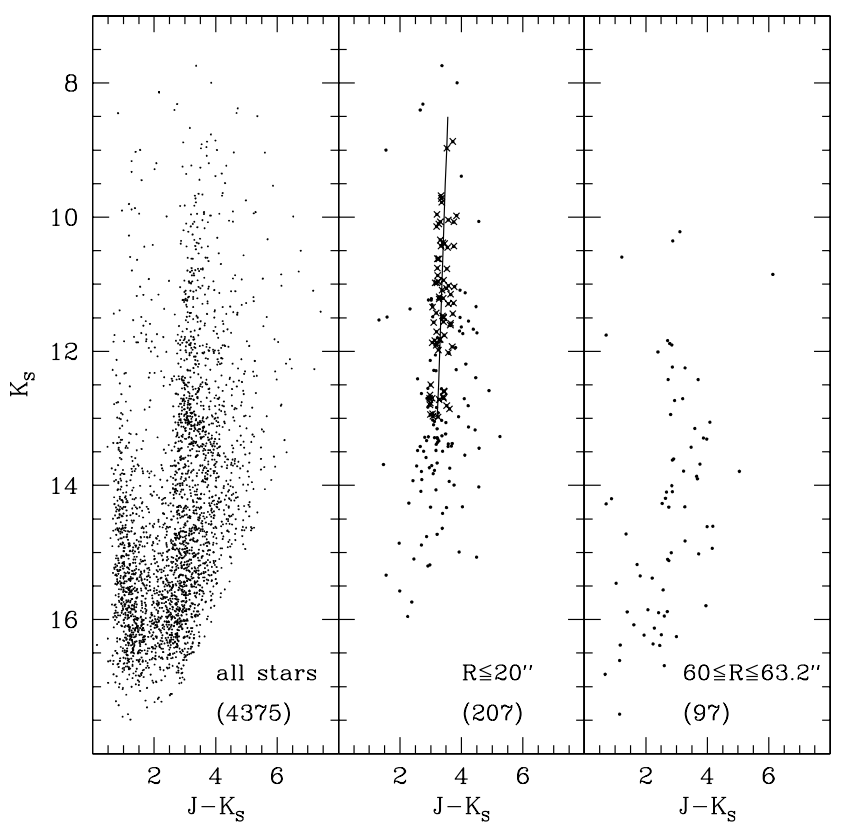

Fig. 4. Near infrared color-magnitude diagram for: all stars in the field of GLIMPSE-C01 (left panel), and for starts in two regions with identical areas - a circle with radius $R=20$ arcsec around the cluster center (middle panel), and an annulus around the cluster with inner radius of 60 arcsec (right panel). The numbers of stars plotted in each panel are given in brackets. In the middle panel: the best fit to the RGB is drawn with a solid line; crosses mark stars included in one realization (see Sect. 3.3 for explanation).

become apparent from the other two panels: the cluster (middle panel) shows a red giant branch (hereafter RGB) at $J-K_{\mathrm{S}} \sim$ 3-4 mag, and the field (right panel) is dominated by main sequence stars at $J-K_{\mathrm{S}} \sim 1-2$ mag and a group of red stars with $J-K_{\mathrm{S}} \geq 3 \mathrm{mag}$, possible highly reddened background objects.

An inspection of the CMD reveals the presence of a clump of stars in the red giant at $K_{\mathrm{S}} \sim 13.0 \mathrm{mag}$. It is also noticeable in the cluster luminosity function (Fig. 2). Taking into account the width of the structure along the $K_{\mathrm{S}}$ axis, we assign an

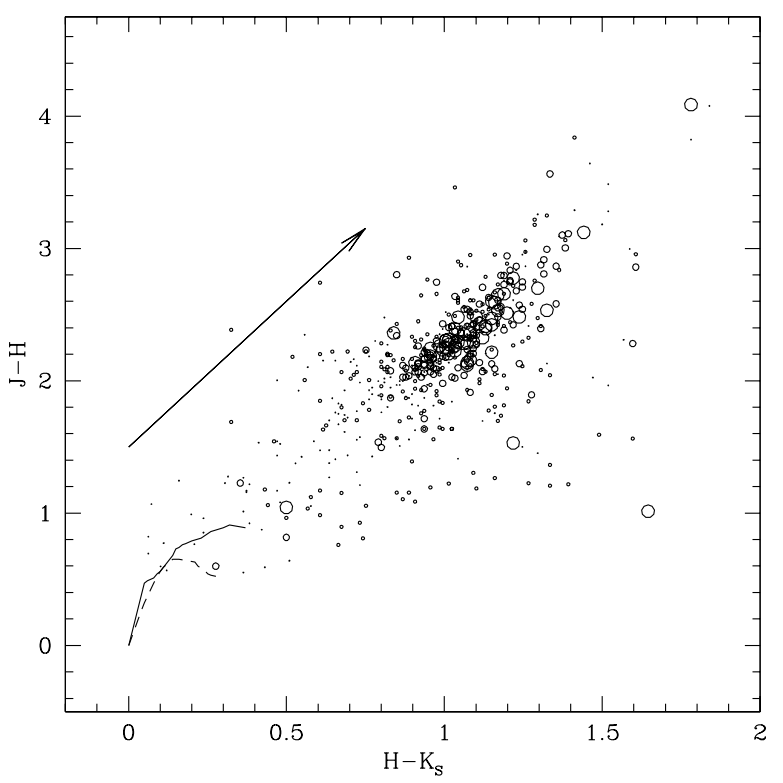

Fig. 5. Near infrared color-color diagram for starts in two regions in the vicinity of GLIMPSE-C01, with identical areas - a circle with radius $R=30$ arcsec around the cluster center (open circles; the size is proportional to the apparent brightness of the star), and an annulus around the cluster with an inner radius of $50 \operatorname{arcsec}$ (solid dots). A vector representing visual extinction $A_{\mathrm{V}}=15 \mathrm{mag}$ is plotted. The colors of RGB (solid line) and the main sequence stars (dashed line) are also shown (Frogel et al. 1978; no correction for the photometric systems is applied).

uncertainty of $0.25 \mathrm{mag}$ (equal to the bin size used to build the luminosity function) to the apparent magnitude of the clump. If this structure is indeed the red clump, it has an absolute magnitude of $M_{K}=-1.61 \pm 0.03 \mathrm{mag}$ (Alves 2000), yielding a distance modulus $(m-M)_{K} \sim 14.6 \pm 0.3 \mathrm{mag}$. Note that this calibration does not take into account any metallicity effects (see for discussion Salaris \& Girardi 2002).

The distance to the cluster can also be measured using the RGB tip (i.e. Ivanov \& Borissova 2002) but this method suffers from a strong metallicity dependence and it is hampered by the small number of stars at the upper end of the RGB. Nevertheless, we carried out this test as a consistency check. If the tip is located at $K_{\mathrm{S}} \sim 8.7 \mathrm{mag}$, and assuming $M_{K}=-6.1 \mathrm{mag}$ for $[\mathrm{Fe} / \mathrm{H}]=-1.5($ Ivanov \& Borissova 2002) we obtain $(m-M)_{K} \sim 14.8 \mathrm{mag}$, in agreement with the estimate given above.

The color-color diagram of the cluster field is shown in Fig. 5. Our data confirm the visual extinction $A_{\mathrm{V}} \sim 15 \pm 3 \mathrm{mag}$ derived by Kobulnicky et al. (2005). Throughout this paper we used the reddening law of Rieke \& Lebofsky (1985), giving $A_{K} \sim 1.7 \pm 0.3 \mathrm{mag}$. This yields a reddening-free distance modulus of $(m-M)_{0} \sim 12.9 \pm 0.4 \mathrm{mag}$, or $D \sim 3.8 \pm 0.7 \mathrm{kpc}$, in agreement with Kobulnicky et al. (2005). The CMD shows a notable spread of colors among the fainter stars that might be contributed to differential extinction and to contamination from extended emission and background sources. 


\subsection{Metal abundance}

The RGB slope allows us to derive abundances of globular clusters (i.e. De Costa \& Armandroff 1990) because of the metallicity-dependent opacities in cool stars. The RGB slope is independent of the reddening, which is an important advantage in studies of heavily obscured clusters such as GLIMPSE-C01. Here we applied the analysis and the calibrations described in Ivanov \& Borissova (2002).

The first step was to remove the fore- and background stars, which constitute $\sim 8-9 \%$ of the stars in the designated cluster area - a circle with 40 arcsec diameter. We used a Monte-Carlo technique: the CMD of the cluster was divided into rectangular bins, as was the CMD of a circular annulus, centered on the cluster, with an inner radius 60 arcsec and the same area as the cluster region. Then, from each bin of the cluster CMD we randomly remove a number of stars, equal to the number of stars in the corresponding bin of the "field" CMD, producing a single realization. Next, we carried out a least square fit to the RGB (Ivanov \& Borissova 2002; see also Appendix A in this work):

$\left(J-K_{\mathrm{S}}\right)=Z P+$ Slope $\times K_{\mathrm{S}}$

on the corrected diagram to derive the slope and the zero point. The RG locus was defined after inspecting the CMD: $8.5 \leq K_{\mathrm{S}} \leq 13.0 \mathrm{mag}$, and $2.5 \leq J-K_{S} \leq 4.0 \mathrm{mag}$. The bin-size was 0.5 mag along both axes. We removed the $10-\sigma$ outliers, and repeated the fitting. Typically, the fitting coefficients obtained in the two iterations were statistically indistinguishable. Note that the spread of colors along the RGB is dominated by differential extinction, rather than measurement errors. A total of $10^{4}$ realizations were obtained, and we averaged the fitting results. The distributions of the RGB slope and the zero point of the fits are shown in Fig. 6.

Finally, we derived the metal abundance from the calibration of Ivanov \& Borissova (2002), and obtained $[\mathrm{Fe} / \mathrm{H}]=$ $-1.61 \pm 0.14$ in the scale of Zinn (as implemented in Harris 1996), $[\mathrm{Fe} / \mathrm{H}]=-1.44 \pm 0.12$ in the scale of Caretta \& Gratton (1997) and $[\mathrm{Fe} / \mathrm{H}]=-1.12 \pm 0.12$ in the scale of Ferraro et al. (1999). The calibrations of Valenti et al. (2004) yield $[\mathrm{Fe} / \mathrm{H}]=-1.14 \pm 0.16$ and $[\mathrm{Fe} / \mathrm{H}]=-0.97 \pm 0.15$ for the last two scales. Both values are systematically lower than ours but the differences are within $1-2 \sigma$.

These measurements rely on the important assumption that GLIMPSE-C01 has the same age as the other Milky Way globular clusters used to derive the metallicity versus RGB slope calibrations. The comparison of the cluster CMD with stellar isochrones (Kobulnicky et al. 2005) seems to indicate an age of at least $8 \mathrm{Gyr}$ but this issue cannot be addressed until a more accurate age estimate becomes available.

\section{The case of the missing clusters}

The discoveries of GLIMPSE-C01 and Whitting-1 prompted us to reinvestigate the question of whether there are any more undiscovered globular clusters in the Milky Way. The total number of Milky Way globulars estimated by van den Bergh (1998) is $160 \pm 20$, slightly above the currently
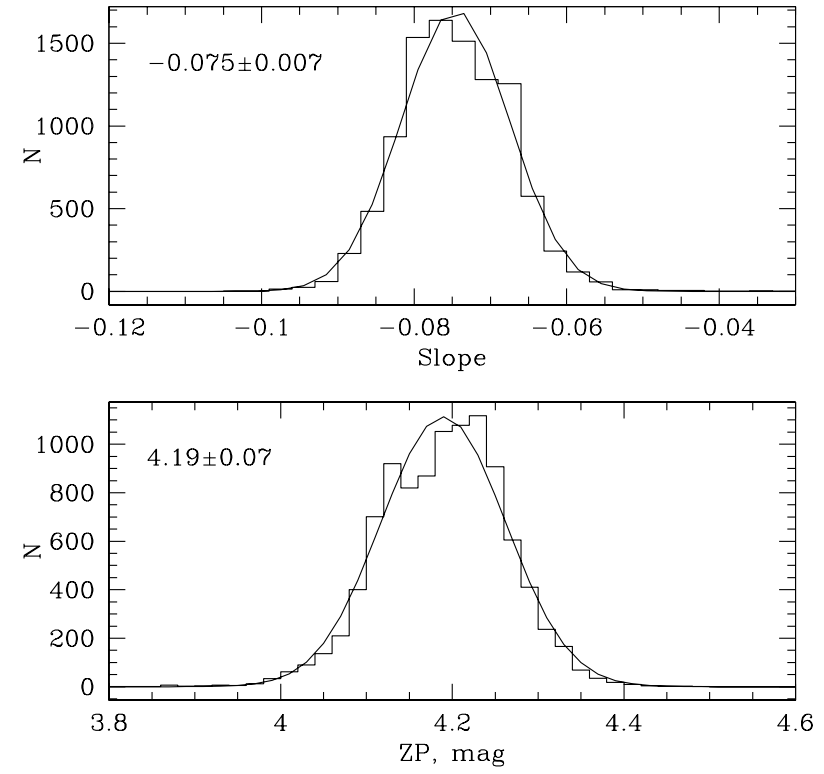

Fig. 6. Distributions of the RGB slope (top panel) and the zero point (bottom panel) of the fits for the $10^{4}$ Monte-Carlo realizations with random removal of the fore- and background contamination. Gaussian fits to the distributions are overplotted, and the average values with their standard errors are given. See Sect. 3.3 for details.

known $\sim 150$ objects. Barbuy et al. (1998; see their Figs. 3 and 4) argued that some missing clusters are probably located in the general direction of the Galactic Center. Here we adopt a modification of their method. The $X Y Z$ Galactic coordinates used in this section are taken from the Feb. 2003 edition of the Milky Way Globular Cluster Catalog (Harris 1996). A possible caveat is the variation of the ratio of the total extinction to the reddening $R_{V}$. For example, a change or $R_{V}$ from 3 to 3.3 introduces a $10 \%$ change in the distance, or almost $1 \mathrm{kpc}$ at the distance of the Galactic Center. The averaging over different dust properties along the line of sight helps to minimize the related distance uncertainties but the variations of $R_{V}$ toward the bulge need further investigation.

First, we assumed that all missing clusters are located in the Galactic plane close to the Galactic Center. Therefore, we consider only the region with $|Z| \leq 0.5 \mathrm{kpc}$ and $R_{\mathrm{GC}} \leq 3.0 \mathrm{kpc}$. These constraints reflect the spatial distribution of the obscuring material. They make our estimate of the missing clusters only a lower limit, as it was demonstrated by the recent discovery of the off-the-plane cluster Whiting-1.

An observer at the Galactic Center should detect an equal number of globulars toward the Sun, in the antisolar direction, and in the directions perpendicular to the line connecting the Sun and the Galactic Center, i.e. the globular cluster distribution along any direction in the Galactic plane should be flat. However, it shows some structure (Fig. 7): there are more clusters in the directions toward the Sun and in the opposite direction than in the direction perpendicular to the line connected the Galactic Center and the Sun. Ten additional clusters are necessary to flatten the histogram (indicated by the shaded area), setting a lower limit to the missing clusters. 


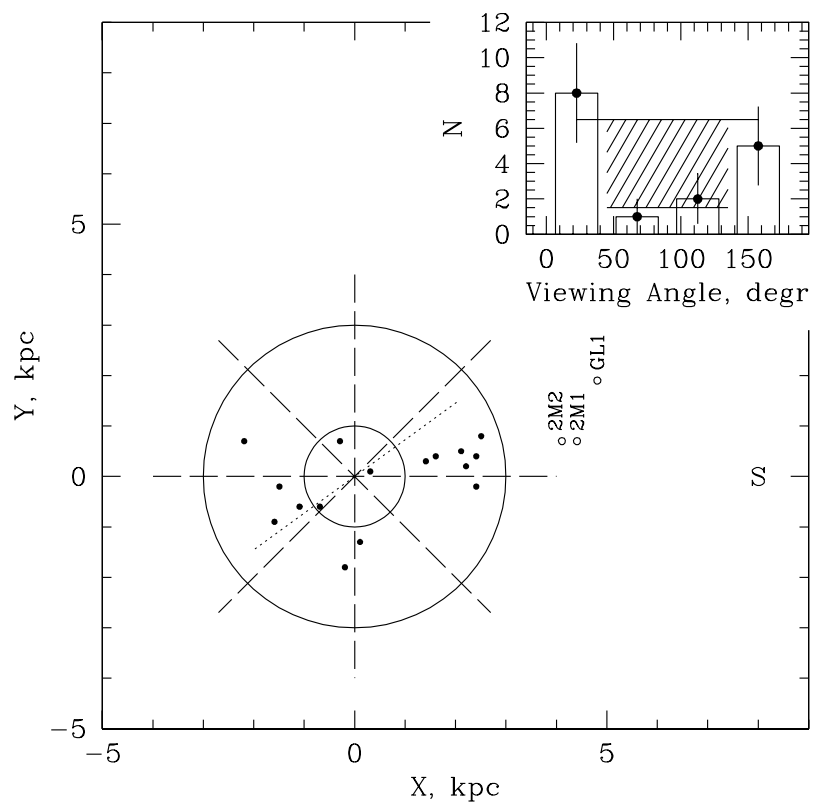

Fig. 7. Spatial location of the globular clusters in the selected region (see Sect. 4) projected on the Galactic plane. The Sun is marked with $\mathrm{S}$, the Galactic Center lies at the origin of the plot. The $X$-axis increases toward the Sun and the $Y$-axis increases in the direction of Galactic rotation. The locations of 2MASS GC01, 2MASS GC02 and GLIMPSE C01 are indicated (2M1, 2M2 and GL1, respectively). The dotted line shows the position of the Galactic bar, according to Weinberg (1992). Dashed lines indicate the limits of the bins for the histogram shown in the inset - it gives the distribution of the number of clusters in the selected region viewed from the Galactic Center. The viewing angle is 0 deg in the direction toward the Sun and it increases to $180 \mathrm{deg}$ in the antisolar direction. Poisson uncertainties are shown with bars. The area of the shaded zone limited by the average level of the outer and the inner bins corresponds to the number of the clusters needed to obtain a homogeneous distribution.

This estimate has to be treated with caution. For example, it is sensitive to the adopted globular cluster distance, and may contain systematic errors related to the adopted globular cluster scale (and the Galactic Center distance). In addition, while the location of the clusters on the sky are known very well, their distances contain statistical observational errors, artificially extending the distribution along the line of sight, similarly to the "finger of God" effect known to extragalactic astronomers. This would move some of the clusters from the middle two bins to the outer bins, increasing the estimate.

The spatial location of the clusters may be affected by the presence of a bar or a triaxial bulge in our Galaxy. The major axis of the bar has an angle of only about $36 \pm 10$ deg from the line of sight toward the Galactic center (Weinberg 1992), with the near end lying in the first quadrant. The major axis of the bar is close to the direction in which the cluster distribution is elongated.

Our result suggests that the searches for hidden clusters carried out so far are biased toward the regions closer to the Galactic Center so the further away a region is from the Galactic Center, the less likely it is to be explored. This implies that the future searches must modify their strategy to include regions in the Galactic plane as far as 7-15 deg from the Galactic Center.

\section{Summary}

We report deep near infrared photometry of the newly discovered Galactic globular Cluster GLIMPSE-C01, and we derived for the first time the metal abundance of this object from the slope of the RGB. The cluster appears metal-poor, with $[\mathrm{Fe} / \mathrm{H}]=-1.6$ in the scale of Zinn. We confirm the distance and reddening estimates of Kobulnicky et al. (2005), placing the cluster at $D \sim 3.7 \pm 0.8 \mathrm{kpc}$, with $A_{\mathrm{V}} \sim 15 \mathrm{mag}$ of visual extinction.

Finally, we estimate number of the missing clusters in the central region of the Milky Way. Based on the location of the known clusters, and assuming radial symmetry of the cluster distribution around the Galactic center, we conclude that the Milky Way contains at least $10 \pm 3$ undiscovered globular clusters.

Acknowledgements. This publication makes use of data products from the Two Micron All Sky Survey, which is a joint project of the University of Massachusetts and the Infrared Processing and Analysis Center/California Institute of Technology, funded by the National Aeronautics and Space Administration and the National Science Foundation. This research has made use of the SIMBAD database, operated at CDS, Strasbourg, France. The authors thank Dr. Ortolani for the useful comments.

\section{Appendix A: Red Giant Slope - Metallicity calibration}

The equations given bellow replace Eqs. (1)-(4) from Ivanov $\&$ Borissova (2002) which contained an error.

$$
\begin{aligned}
\left(J-K_{\mathrm{S}}\right)_{0}= & R G B_{\mathrm{ZP}}+R G B_{S l} \times M_{K_{\mathrm{S}}} \\
R G B_{S l}= & a_{0}^{S l}+a_{1}^{S l} \times[\mathrm{Fe} / \mathrm{H}] \\
R G B_{Z P}= & a_{0}^{\mathrm{ZP}}+a_{1}^{\mathrm{ZP}} \times[\mathrm{Fe} / \mathrm{H}] \\
{[\mathrm{Fe} / \mathrm{H}]=} & {\left[\left(J-K_{\mathrm{S}}\right)_{0}-a_{0}^{S l} \times M_{K_{\mathrm{S}}}-a_{0}^{\mathrm{ZP}}\right] / } \\
& {\left[a_{1}^{S \mathrm{p}} \times M_{K_{\mathrm{S}}}+a_{1}^{\mathrm{ZP}}\right] }
\end{aligned}
$$

\section{References}

Alves, D. 2000, ApJ, 539, 732

Barbuy, B., Bica, E., \& Ortolani, S. 1998, A\&A, 333, 117

Benjamin, R., Churchwell, E., Babler, B. L., et al. 2003, PASP, 115, 953

Borissova, J., Pessev, P., Ivanov, et al. 2003, A\&A, 411, 83

Borissova, J., Ivanov, V. D., Minniti, D. Geisler, D., \& Stephens, A. W. 2005, A\&A, 435, 95

Carraro, G. 2005, ApJ, 621, L61 
Carretta, E., \& Gratton, R. G. 1997, A\&AS, 121, 95

De Costa, G., \& Armandroff, T. 1990, AJ, 100, 162

Fazio, G., Hora, J. L., Allen, L. E., et al. 2004, ApJS, 154, 10

Ferraro, F. R., Messineo, M., Fusi Pecci, F., et al. 1999, AJ, 118, 1738

Frogel, J., Persson, S. E., Matthews, K., \& Aaronson, M. 1978, ApJ, 220,75

Ivanov, V. D., \& Borissova, J. 2002, A\&A, 390, 937

Ivanov, V. D., Borissova, J., Pessev, P., Ivanov, G.R., \& Kurtev, R. 2002, A\&A, 394, L1

Ivanov, V. D., Borissova, J., Bresolin, F., \& Pessev, P. 2005, A\&A, 435,107

King, I. 1962, AJ, 67, 471
Harris, W. E. 1996, AJ, 112, 1487

Hurt, R. L., Jarrett, T. H., Kirkpatrick, J.D., et al. 2000, AJ, 120, 1876 Kobulnicky, H. A., Monson, A. J., Buckalew, B.A., et al. 2005, AJ, 129,239

Rieke, G. H., \& Lebofsky, M. J. 1985, ApJ, 288, 618

Salaris, M., \& Girardi, L. 2002, MNRAS, 337, 332

Simpson, J. P., \& Cotera, A. S. 2004, BASS, 204, No. 45.01

Stetson, P. B. 1993, User's Manual for DAOPHOT II

Valenti, E., Ferraro, F. R., \& Origlia, L. 2004, MNRAS, 351, 1204

van den Bergh, S. 1998, A\&A Rev., 9, 273

Weinberg, M. D. 1992, ApJ, 384, 81 